\title{
Study on the Characteristics and Aesthetics of Chinese Ceramic Lamps
}

\author{
Mei Liao \\ Panzhihua University \\ Panzhihua, China
}

\begin{abstract}
In the historical process of succession of Chinese civilization, ceramic lamps as a kind of cultural treasures with long history have decisive influence in the development of contemporary Chinese civilization. Based on its own characteristics, this paper demonstrates its superiority in lamp design from the aspects of plasticity, safety and structure. From the artistic point of view, with the historical changes and technological development, the paper analyzes the aesthetic value of ceramic lamps from different angles.
\end{abstract}

Keywords—ceramic lamps; characteristics; aesthetic value

\section{INTRODUCTION}

Lamps as an indispensable tool in the daily life of modern people are closely related with aesthetics and living standards of people. Among the different making materials of the lamps, ceramics occupy an important position in the development of Chinese arts and crafts based on their unique materials, techniques and shapes. Throughout the ages, the proportion of ceramic lamps in the field of people's lives and aesthetic area are relatively large. The combination of lighting art and decorative design has formed a high degree of unity. This article discusses the development of China's ceramic lamps in the development of the design. And the summary is shown as the following.

\section{CHARACTERISTICS OF CHINESE CERAMIC LAMPS}

\section{A. The Plasticity}

First of all, from the perspective of the material of ceramic lamps, the main molding material is clay. Through the operation, it can form different shapes and texture. We can see that it has strong plasticity. Second, the ceramic has the characteristics of convenient processing and large potential dimension. In the case of different glazes, the artistic appeal is also quite different. With the unconstrained lamp design, the combination of the two sides can maximize the display of the activity of light and shadow [1]. In addition, it is mainly formed through the interaction between mud and fire from the point of view of the formation of ceramic. It is not only more affinity in the material, but also is simple in the shape. Thus, it would enhance the artistic appeal of ceramic lamps.

\section{B. The Security}

Ceramic lamps could be used in public space. And its unique high-temperature resistance is a remarkable advantage. As mentioned above, ceramics is a kind of handicraft which is formed by the blending of mud and fire. It means that its relatively high-temperature resistance could prevent the occurrence of odor in long-term use. It also could avoid the risk of becoming flammable. On the other hand, with the strong electrical conductivity of ceramics, they are often used as an insulating material. Thus, it provides further level of protection for the safety of ceramic lamps.

\section{The Structural Rationality}

In view of the uniqueness of Chinese ceramic lamps in the artistic expression, we can see that the shaping of their structure is a reflection of the decorative performance, which has aroused people's interest in the structural rationality. In the modeling design of ceramic lamps, people not only pay enough attention to the balance and stability of their shapes, but also make some efforts to improve their structural rationality. People mainly combine their practicality with the times together, and carry out scientific unification. As a result, we should promote its stylistic features to be brought into full play. And then, the value of artistic expression can be enhanced.

\section{The Aesthetic VAlue OF Chinese CeRAMIC LAMPS}

\section{A. The Material}

From the point of view of components of the ceramic, ceramic body, glaze and decorative materials are the main components. With the role of dozens of processes, several factors have formed the ceramic art works. The fixed base, lampshade, light source and other parts constitute the ceramic lamps. The base part as the most commonly involved in the ceramic material has the flexibility, diversity and other characteristics. Also, it has a greater development in artistic expression. In the continuous development of ceramic technology, in addition to the lamp base, the ceramics is also gradually applied to the lampshade design. It has formed a certain role in promoting the development of the design of the ceramics. Material as an important symbol of ceramic art value, has occupied an important artistic status since ancient times. Taking celadon glaze as an example, $\mathrm{Lu}$ Guimeng made a targeted description of its material 
characteristics with the phrase of "winds in September turned over Yue ware, and the porcelain was beautiful". And we can see his admiration for the artistic features. What is worth mentioning, due to the profound development of ceramic lamps in China and different cultural characteristics of the dynasties, it has formed certain differences of ceramic lamps in the cultural accumulation. [2] For example, from the characteristics of ceramic lamps in late Tang dynasty, the porcelain body is in the subtle state. And the design tends to be more refined. We should handle the porcelain with glazing the whole body as the main method. The glaze is in shiny state. During this period, part of the ceramic lamps and lanterns will be hollowed out in the artifacts. And they would also make the printing and other processing on the ceramic lamps and lanterns. Based on the original shape, we could add finishing touch. Therefore, it would further enhance the visual impact. During the Five Dynasties Period, we could realize something about ceramic lamps. For example, the white porcelain of Jingdezhen was the mainstay of the ceramic industry during the period. And the porcelain has the reputation of "fake jade". The material of the porcelain was clear and bright, which was the main features of the porcelain. With unique artistry, we could produce special ceramics porcelain. At the same time, we could add the deep culture of porcelain. And it has provided important technological conditions for the further development of porcelain lamps.

All in all, the artistic expression and cultural heritage of ceramic lamps are inseparable from the unique material. The selection of the material can also play a decisive role in its actual aesthetic value. Therefore, with the continuous development of ceramic technology, we can still regard its material screening as an important way of development. From an artistic perspective, we could combine the traditional ceramic technology with modern innovative technology organically. Therefore, it would achieve the practicality of ceramic lamps and aesthetic value.

\section{B. The Craft}

In addition to the material, the application of ceramic decoration technology is also an important factor for its aesthetic value. In terms of the existing conditions under the artistic environment, there are many kinds of related arts and crafts applied to the decoration of ceramic arts. Commonly, there are paintings, sculptures, hollows, etc. In the case of ingenious fusion with the original shape of the lamps, it would not only enrich the visual perception of people, but also could promote the diversity of decorating techniques of ceramic lamps. [3] Now, we could take thin-layer ceramic lamps as the example, which is common in the current ceramic lamp market. At present, this tool has strong mainstream. And it mainly originated from the Northern Song dynasty. At that time, the thin-layer ceramic had embryonic shape. It was known as the shadowy blue ware. From the characteristics of shadowy blue ware, it is often relatively delicate in the processing of porcelain. In terms of glaze processing, it would avoid the limitations of single color. The color of shadowy blue ware mostly is bluish white. And in the traits, the shadowy blue ware is shiny. And it is known as "prime muscle jade bone". In addition, with the uniqueness of shadowy blue ware in forming shape, it often requires dozens of strict processes such as selecting materials, beating, molding, repairing blanks, burning kilns, and so on. To a large extent, it could meticulously display the aesthetic value of ceramic craft. Ceramic table lamp is the main product with the thin-layer technology. Through the combination of textured base and thin-layer ceramic lampshades, it has formed unique artistic perception, which would provide people with better visual and environmental experience.

Also, we could take another mainstream technology hollow ceramic lamps as the example. Compared with the thin-layer ceramic lamps, hollow ceramic lamps have a long history of development since the more ancient era. In the early stages of development of the hollow ceramic, its production process has tremendous difficulty. For example, the porcelain workers should make processing with a blade directly on the body. And then, it would show us the riceshaped porcelain. In addition, we should seal up the riceshaped hole with the glaze sealing process. Based on the application of its meticulous process, it was known as "delicate eye". [4] In the overall production process of hollow ceramic lamps, each link is in interlinked state. Therefore, if there is a link in the trouble, it has to cancel the overall production process. From the perspective of the process characteristics of hollow ceramic lamps, porcelain workers can exquisitely make clever fusion processing of the blue and white. Then, it would combine the blue with the white. It can form the blue and white exquisite porcelain. In the blending of two colors of green and turquoise, it can give people a sense of elegant visual enjoyment. On the other hand, based on the characteristics of the high-temperature firing of ceramic lamps, Ceramic light is basically opaque. When the light shines through the ceramic lamps, the light would shine from the exquisite eye. Then, the porcelain can present soft and mottled artistic visual effects, which would make people feel more comfortable.

In addition to the above two kinds of ceramic technology, hand-painted process is the major feature in the development of ceramic lamps. And it would also have pivotal impacts on the overall development of ceramic lamps. As the representative of Jingdezhen ceramics lamps, various schools of artists would directly draw various patterns of different shapes on the ceramic lamps. On the basis of original shapes, it would have more unique color and charm. To a certain extent, it would highlight the theme of the lamp. And it can also play visual role of finishing touch. From the perspective of main categories of hand-painted craft, it can be divided into two categories of glaze and underglaze, which includes the glaze color, new color, etc. The underglaze contains high-temperature color glaze, blue and white, etc. In the case of different characteristics of the ceramic, it could adopt different types of hand-painted technology in different situations. With the integration of the two different art forms of painting and ceramic, the actual artistic effects of painting and ceramic can be maximized. Therefore, the fundamental promotion of the development of ceramic lamps can be realized. 
All in all, the molding effect of ceramic lamps, and the characteristics of the idea need to be conveyed through the decorative process, which means that in the course of the development of ceramic lamps, we should choose the screening. And the decorative process would provide important support for the enhancement of art value of the ceramic lamps. In addition, the engraving, sculpture and other processes not mentioned in detail above also play an important promoting role in the development of ceramic lamps. And it can be further studied in the future design of ceramic lamps.

\section{The Artistic Conception}

From the perspective of Chinese traditional aesthetics, it is necessary to give the thing a sense of agility from the perspective of artistic conception. In addition, it should carry out detailed carving of its materials and craftsmanship when it comes to artistic processing. Thus, it completed the overall construction process of the integration of virtuality and reality. Artistic conception, as a kind of relative abstract conceptual form, is mainly derived from the blending of virtuality and reality. Artistic conception belongs to more advanced realm. Based on the above principle, during the design process of ceramic lamps, the real feeling and artistic conception of ceramic lamps can be reflected in the form of "blending emotion into the view". Thus, it would form unique light and shadow art. For example, in modern home decoration, we could use the ceramic lamps to make decoration. It can be dealt with in the form of light. With the soft light and shade, it would create a warm space in the interior. Thus, it would show unique cultural art conception of home environment. With the action of the light, not only can the overall environmental effect of the indoor environment be changed, but also the material and technology of the ceramic lamp can form a kind of complementary and practical effect. Therefore, it could promote the artistic conception of overall space. In the case of different styles of decoration, it can make the appropriate treatment to display the light of ceramic lamps. And then, the lighting efficiency would be in accordance with the actual decoration style. On the other hand, in the process of displaying the ceramic lamps, in order to maintain the overall coordination of the decoration, it should consider and discuss the actual space, decoration materials and other practical factors. And then, it would fundamentally avoid the strangeness of overall visual effects. Taking the schoolroom as an example, when we deal with such space with relatively heavy cultural atmosphere, we can consider the theme of blue and white lighting as the optimization. And it would form an overlap with the overall artistic conception of the study. Viewing from the characteristics of the blue and white ceramic lamps, its color tone processing tends to be blue and white. These two colors add radiance and beauty to each other. The gradation is distinct. The visual sense is relatively natural. And it would form mutual collocation with the study. In the cultural atmosphere created by the blue-and-white porcelain lamps and study, people not only enjoy the comfortable artistic experience, but also feel the infinite mood in a limited space environment. In addition to the values of the pattern listed above, the blue-and-white porcelain lamps also have ornamental value in the context of the contrast. When we turn on the blue and white porcelain lamps, the lower end of the lamp would spread the light. And it would create the light and shadow. At the same time, it also can directly point out the theme of space. People would completely indulge in the artistic atmosphere. It could make the design of ceramic lamps from the artistic level. During this process, it should make key processing on the light and shade of the light, strength and other factors. If necessary, it should also consider the appropriate conversion of light. And then, it would promote the lighting color.

In short, artistic conception, as an important factor involved in the development of artistic aesthetics, can be used as a breakthrough point in the development of Chinese ceramic lamps. The artistic conception of porcelain lamps can be fundamentally enhanced by the combination of specific materials such as the material and technology of ceramic lamps. In addition, we could use ceramic lamps to create artistic conception. However, we should fully comply with the decorative principle of "serving the overall style". We should enhance the overall aesthetic taste. Also, it can't be inconsistent with the overall style. Then, it would avoid counter-productive design work.

\section{CONCLUSION}

In summary, Chinese ceramic lamps have a long history. And it also has a long history in the design and development. In different periods, Chinese ceramic lamps have unique accumulation. Also, it has an important artistic status and historical status. In the current environment, ceramic lamps not only symbolize the symbol of modern home culture, but also serve as a kind of sensory enjoyment under the aesthetic art. The diversification of ceramic lamps is of great value to the construction of high-quality life and the expansion of diverse space. In the future design and development, it would be more flexible and changeable.

\section{REFERENCES}

[1] Rao Huajun, Hu Zhengjuan. Innovative design of Jingdezhen delicate ceramic lamps $[\mathrm{J}]$. Jingdezhen Ceramic, 2013 (02): 8-9.

[2] Zhang Ruixia. To explore the innovative design of the combination of ceramic lamps and clock [J]. Chinese Ceramic, 2012,48 (09): 28-30.

[3] Wang Nannan. The design features of Chinese ancient hand-ceramic lamps [J]. The Science Education Article Collects, 2013 (10): 152153.

[4] Zhang Lizhao. The influences of consumer culture on the design and development of ceramic lamps [J]. Art Science and Technology, 2014 (03): 227. 